\title{
El síndrome de ovario poliquístico: aspectos psicológicos
}

\author{
César Andrés Gómez-Acosta, MSc ${ }^{1 a}$, Stefano Vinaccia Alpi, PhD $2 a$, Japcy Margarita \\ Quiceno, PhD. ${ }^{3 a}$
}

${ }^{1}$ Fundación Universitaria Sanitas. ${ }^{2}$ Grupo de Investigación Psychology and Health Sanitas, Fundación Universitaria Sanitas. ${ }^{3}$ Universidad de San Buenaventura. Colombia.

$\mathrm{a}_{\text {Psicólogo/Psicóloga. }}$

\section{RESUMEN}

Antecedentes: La gran mayoría de la literatura científica sobre el síndrome de ovario poliquístico (SOP) ha utilizado un enfoque bio-médico para analizar esta enfermedad. En América Latina, y específicamente en idioma español, hay escasa información sobre las relaciones del SOP con factores psicológicos. Objetivo: Analizar este trastorno ginecológico de origen endocrino y su asociación con diferentes factores psicológicos, así como su abordaje integral. Método: Se realizó una búsqueda en bases de datos en los últimos años con los términos "SOP", "calidad de vida", "ansiedad", "depresión", "imagen corporal" y "disfunción sexual". Resultados: EI SOP es una enfermedad multifactorial que se ha vinculado a la depresión, la ansiedad, el estrés, la insatisfacción de la imagen corporal, trastornos sexuales y de la alimentación, el funcionamiento cognitivo, calidad de vida y el bienestar psicológico; la co-ocurrencia de este tipo de situaciones psicológicas alimenta la condición somática en mención. Conclusión: El abordaje psicológico puede complementar las acciones preventivas y terapéuticas que influyen en la efectividad del tratamiento y el bienestar subjetivo informado por los pacientes.

\section{PALABRAS CLAVES: Ovario poliquístico, emociones negativas, imagen corporal, trastornos de la alimentación, calidad de vida}

\section{SUMMARY}

Background: The vast majority of the scientific literature on polycystic ovary syndrome (PCOS) have used a bio-medical approach to analyzing this disease. In Latin America, specifically in Spanish, little is known about the relationship of PCOS with psychological factors. Aim: To analyze this gynecological disorder of endocrine origin and their association with different psychological factors and their comprehensive approach. Method: A search was performed in databases in recent years with the terms "PCOS", "quality of life", "anxiety", "depression", "body image" and "sexual dysfunction." Results: PCOS is a multifactorial disease that has been linked to depression, anxiety, stress, dissatisfaction with body image, sexual and eating disorders, cognitive functioning, quality of life and psychological well-being; the co-occurrence of such psychological situations feed the somatic condition analyzed. Conclusion: The psychological approach can complement the preventive and therapeutic actions that influence the effectiveness of treatment and subjective well-being reported by patients.

KEY WORDS: Polycystic ovary, negative emotions, body image, eating disorders, quality of life 


\section{INTRODUCCIÓN}

El síndrome de ovario poliquístico (SOP) se ha convertido en un grave problema de salud pública, siendo el más común de los desórdenes endocrinos en mujeres en edad reproductiva (1), con una proporción de una de cada cinco personas en esta población (2). Es una enfermedad de etiología multifactorial, comórbida en muchos casos con la obesidad, la dislipidemia, los desórdenes cardiovasculares, la hiperplasia endometrial, los desórdenes del periodo menstrual e infertilidad (3), el cáncer endometrial (4), el cáncer de mama (5), y procesos pro-inflamatorios (6); esta condición, a su vez, afecta la tolerancia a la metabolización de la glucosa (7), la resistencia a la insulina y potencia el hiperandrogenismo (1).

Dichos síntomas somáticos suelen precipitar problemas psicológicos entre los que se encuentran estrés, ansiedad, depresión, disminución de la calidad de vida, cambios en la imagen corporal e identidad, baja autoestima, y disfunciones psicosexuales (8), así como la ejecución de prácticas poco saludables en comparación, por ejemplo, con pacientes diabéticos (9); es más, dichas afectaciones no solo se relacionan con el padecimiento de la enfermedad misma, sino también con temores a los riesgos metabólicos y cardiacos, y a la infertilidad $(1,10)$. Sin embargo, a pesar de todo lo señalado, la valoración, el diagnóstico y el tratamiento de los padecimientos psicológicos asociados al SOP, son aún bastante discretos (10), particularmente en los contextos hispanoparlantes.

El objetivo de esta investigación es presentar una revisión sistemática tanto de las evidencias empíricas documentadas respecto a los factores psicológicos que se han demostrado como comórbidos con el SOP, como de su abordaje integral.

\section{MATERIAL Y MÉTODO}

Con la metodología de investigación documental, se abordan 54 artículos publicados desde el 2002, procedentes de las bases de datos MedLine, PsyArticles, Proquest, ScienceDirect y SpringerJournal. Se usaron como palabras clave "síndrome de ovario poliquístico", "calidad de vida" "ansiedad", "depresión", "autoimagen corporal" y "disfunciones sexuales".

\section{RESULTADOS}

Estrés. Se identifica el estrés con frecuencia en las mujeres jóvenes con SOP, que resulta potencializado indirectamente el aumento de la testosterona vía hipotálamo-pituitario-adrenal (HPA) $(12,13)$, contribuyendo a la presencia de hirsutismo y otras implicaciones estéticas (14); esto reduce significativamente la identidad femenina, y por esta vía, se retroalimenta en la forma de distrés psicológico (15), y de disfunciones sexuales $(16,17,18)$. Asimismo, el estrés compromete negativamente la regularidad del ciclo menstrual y de la ovulación, incidiendo en fuertes cambios endocrinos y emocionales (16).

Al comparar con sujetos controles, las pacientes con SOP presentan mayor actividad de ACTH plasmático y corticoides por sobre exposición a situaciones estresantes inducidas (11). No hay que olvidar que el cortisol producido durante periodos de distrés crónico induce, mediante mecanismos epigenéticos, el incremento del riesgo cardiovascular y de infarto de miocardio (11), reportado a partir del aumento de la presión sistólica y diastólica, así como de la activación exacerbada de citoquinas pro-inflamatorias (19). Estas últimas, en conjunto con el estrés, potencian cambios comportamentales relacionados con anhedonia, fatiga, movimientos retardados, trastornos alimentarios, aislamiento social, desórdenes del sueño y distorsiones cognitivas, que a su vez repercuten en mayor vulnerabilidad a los estados depresivos, y en aumento a la sensibilidad al dolor. Además, una vez que las citoquinas pro-inflamatorias atraviesan la barrera hemato-encefálica, producen alteraciones en la síntesis de serotonina y norepinefrina, claves en el desarrollo de los estados depresivos (9).

Las pacientes con SOP tienden a tener un eje HPA hiperrespondiente ante las acontecimientos amenazantes, lo cual puede asociarse con mayor predisposición a patologías como depresión, riesgos cardiovasculares y diabetes tipo II (11), a su vez relacionadas con comportamientos insalubres, el impedimento de la adherencia a los tratamientos, mayor predisposición a la obesidad y a la resistencia a la insulina, así como el incremento de la inflamación crónica (11).

Autoimagen corporal. La autoimagen corporal corresponde a la relación entre la silueta y los significados otorgados culturalmente al estar saludable, atractivo y apto para la reproducción (11). La percepción de belleza y atractivo físico en las culturas occidentales está basada en modelos delgados, siendo distinta a los cánones establecidos por los pueblos orientales, en los que incluso se percibe al sobrepeso como signo de prosperidad y estética (11). Al interior de cada cultura, hombres y mujeres buscan que su imagen corresponda con los estándares impuestos, y cuando esto no sucede surgen dificultades en la autoimagen y la autoestima, que a su vez, repercuten negativamente en su salud integral (20), en la medida en que se convierte en condición estresante, que potencia la adopción de prácticas riesgosas, particularmente por las pacientes más jóvenes, en un nivel similar al de las mujeres jóvenes sin SOP (21).

Preocupan bastante las ambivalencias que emergen con la identificación del género, asociadas 
a la edad y a la severidad de las manifestaciones clínicas, lo que crea conflicto con los esquemas femeninos impuestos culturalmente, y potencia roles sociales inadaptados (22). Los cambios que se van presentando en la figura corporal también inciden en la calidad de vida percibida (23), representando para las pacientes con SOP una menor satisfacción con su cuerpo, y una pobre autoimagen en las mujeres que presentan además hirsutismo y sobrepeso $(24,25)$. De hecho, las mujeres con SOP señalan que un mayor deterioro de la calidad de vida se debe mas a los cambios en la apariencia física y al hirsutismo, que a los cambios metabólicos y endocrinos asociados a la irregularidad menstrual e infertilidad (26).

Por otra parte, en contraste con el grupo control, las pacientes con SOP se atribuyen mayor autoculpa e impotencia, así como menor aceptación de la enfermedad, lo que se traduce en dificultades para afrontar adecuadamente los desafíos estresantes a los cuales se ven expuestas, y a su vez, potencia el riesgo de desarrollar desórdenes psicosomáticos futuros (27).

Otros autores (28) también encuentran que las mujeres con SOP presentan tendencia a rasgos de personalidad neuróticos, más dificultades en el control de la ira, y señalan mayor deterioro de su calidad de vida en relación con las mujeres del grupo control, registrando un $70 \%$ de las pacientes indagadas rasgos de personalidad irritable (29). De igual manera, se encuentra que las pacientes con SOP que presentan un Índice de Masas Corporal (IMC) mayor a 25 evidencian menor tolerancia al estrés (13).

Por último, se encuentra que las mujeres con SOP sin sobrepeso presentan una fuerte asociación entre la percepción distorsionada de su imagen corporal y la severidad del trastorno depresivo concomitante (30).

Depresión. Las personas con SOP tienden a presentar depresión, aunque con bastante heterogeneidad entre los estudios comparados $(13,31)$, que incluyen grupos con y sin medicación anti-andrógena, así como mujeres con un alto IMC, siendo este último perfilado como el factor de riesgo más relevante al respecto (13). No obstante, las diferencias en la depresión manifestada por pacientes y grupo control son significativas a nivel estadístico, más no a nivel clínico (13).

Una imagen corporal distorsionada, las actitudes negativas y la autoestima, afectan el estado de ánimo de las personas con SOP (9), incluso, potenciando desórdenes alimentarios, sufrimiento físico, baja productividad, e ideación suicida, con relación al resto de la población femenina $(9,16,32)$. El sentirse poco atractivas induce una menor frecuencia de actividad sexual y disminución de la autoestima, con consecuencias emocionales negativas en sus relaciones interpersonales y de pareja (20). Adicio- nalmente, la depresión es un poderoso factor psicosocial que potencia mayor riesgo cardiovascular (32) y aumento en la producción de interleucina IL6 , que a su vez conlleva a una mayor predisposición hacia la inflamación y dolor de los tejidos $(6,33)$.

Las mujeres con SOP presentan anovulación crónica asociada con bajas tasas de embarazo, infertilidad y bajo estado del ánimo asociado a dicha condición $(18,34)$. Así, la infertilidad es la consecuencia del SOP que más se ha relacionado con la depresión (16); no obstante, estos hallazgos deberán estar sometidos a mayor comprobación (7).

Las pacientes jóvenes son más proclives a sufrir cambios notables en el estado del ánimo en relación con la insatisfacción de sus necesidades emocionales, como son la falta de comprensión de la enfermedad, la forma de afrontar los cambios físicos concomitantes, y la frustración derivada del bullying al cual son sometidas por sus grupos de referencia (35).

Aunque es evidente que la depresión es la condición psicológica que más afecta a las mujeres con SOP (36), esta tiende a disminuir hasta en un $20 \%$ por cada año de educación certificado, de tal manera que quienes tienen mayor nivel educativo demuestran menor afectación de su estado del ánimo (37), elemento que puede ser fundamental en el abordaje preventivo integral. Por otra parte, en algunos tratamientos farmacológicos, se han utilizado pastillas anticonceptivas para reducir la insulino resistencia, con efectos colaterales como la reducción de la tendencia depresiva en las pacientes (12).

Ansiedad. Se encuentran niveles altos de ansiedad en pacientes con SOP con y sin alto IMC $(10,13)$, aun cuando dicha tendencia es mayor para el primer grupo referenciado (38), sugiriendo que a mayor IMC es mayor el riesgo para desarrollar estados de ansiedad (10). A nivel fisiológico, se identifica una correlación entre altos niveles de grelina plasmática (a su vez, asociada con disfunciones del hipotálamo primario) y ansiedad en dichas pacientes (13), pero también se evidencia que niveles altos de ansiedad correlacionan con alta resistencia a la insulina y con la hiperandrogenia, independientemente de la edad y del IMC (39). Se sostiene que la ansiedad es uno de los factores que más afecta la calidad de vida en mujeres con SOP (16), y se identifica que dicha condición aumenta con relación a la población sana (40), quizás por la presión familiar y social a la que son sometidas (16).

La ansiedad, independientemente de su identificación como rasgo o como estado, es significativamente más alta en pacientes con SOP, correlacionando con la alta resistencia a la insulina. EI diagnóstico del SOP en mujeres está relacionado con el incremento de la ansiedad generalizada (31) y la ansiedad social (41), que compromete el funcionamiento en sus contextos familiar, laboral y 
relacional. Se documenta además que cerca de un tercio de las mujeres diagnosticadas con SOP presentan ansiedad, y un 15\% reportan problemas de ansiedad y depresión co-mórbidas (32).

Funcionamiento cognitivo. Las mujeres con SOP presentan, en relación con las controles, compromisos en la resolución de tareas cognitivas, específicamente en la precisión y velocidad de respuesta en pruebas de reconocimiento de palabras (42), más no presentan diferencias en tareas que implican rotación y ubicación espacial (43). Las pacientes no tratadas con anti-andrógenos no mejoran su ejecución en tareas matemáticas y visoespaciales por presentar altos niveles de testosterona, con relación tanto a las mujeres control como a quienes padeciendo SOP recibieron este tipo de medicación; en otras palabras, la presencia de una condición híperandrogénica en las pacientes con SOP no masculinizó sus patrones de funcionamiento cognitivo.

Se ha encontrado una relación estadística entre la presencia de rasgos autistas, depresión, ansiedad (44) y problemas de comunicación en mujeres con SOP, en contraste con sujetos control, condición probablemente asociada a que ambas patologías comparten una presencia de andrógenos elevada en la etapa fetal (2). Otro estudio encontró diferencias significativas en los puntajes obtenidos en una prueba para medir coeficiente intelectual entre las pacientes con SOP y las pacientes del grupo control, aunque los autores señalan que no se puede concluir que dicha distinción obedezca a síntomas específicos de la enfermedad o acambios en el estado de ánimo (12).

Calidad de vida relacionada con la salud (CVRS) y bienestar psicológico. Si bien la CVRS es un concepto relativo en función de las expectativas relacionadas con características como el sexo, la edad y la idiosincrasia socio-cultural particular (45), para el caso de las pacientes con SOP, se evidencian niveles bajos de CVRS en relación con los sujetos control $(6,15)$. El exceso de peso, la amenorrea, el hirsutismo y el acné se vinculan como factores que afectan la autoestima, la imagen corporal, el afrontamiento ante situaciones de estrés y el desarrollo de una sexualidad normalizada (45), y por ende, contribuyen a reducir la CVRS de estas personas $(10,46)$, en una espiral creciente (16), dado que a mayor afectación clínica, mayor deterioro de la CVRS. De acuerdo con la evidencia, se afirma que el exceso de peso es la condición que más afecta la CVRS $(34,47)$.

Otro aspecto que afecta la CVRS de las mujeres con SOP es la demora en el diagnóstico y la percepción de ineficacia de los tratamientos (48), aun cuando no hay una relación directa entre la severidad clínica del problema y la calidad de vida reportada (44).

Al comparar pacientes con SOP y controles, se encuentra que las primeras tienen bajos niveles de CVRS en las dimensiones de infertilidad, emociones y percepción del peso (31), así como mayor insatisfacción con su vida $(5,49)$, aunque algunos autores refieren que no existen diferencias significativas entre pacientes con sobrepeso y delgadas (17), ni en los niveles de CVRS reportada entre las pacientes con SOP en hirsutismo, obesidad e infertilidad (19)

Las mujeres pre-menopáusicas con SOP reportan menores puntajes en el área emocional con relación a las sujetos control, pero estás últimas refieren menor tolerancia al dolor (50). De igual forma, al comparar a pacientes con SOP con pacientes de diabetes tipo II, epilepsia, desorden coronario, dolor crónico, asma, artritis y epilepsia, se encuentra que quienes sufren de SOP refieren igual o incluso mejor CVRS en el área física, más no en su salud mental (51).

\section{MANEJO TERAPÉUTICO}

El manejo del SOP implica enfocar en aspectos como el soporte bio-psico-social (7), la educación preventiva, y el direccionamiento de factores psicológicos para un mejor estilo de vida, involucrando esencialmente cambios significativos en hábitos como la dieta y la realización de actividad física prescrita con su correspondiente acompañamiento profesional para garantizar la pérdida de peso (1). En esa medida, el tratamiento debe ser integral, incluyendo consejería psicológica y participación en grupos de ayuda que permitan construir alternativas efectivas de afrontamiento a las diversas condiciones que afectan la calidad de vida en estas pacientes.

Es curioso señalar que a pesar del distrés psicológico y el deterioro en la calidad de vida en salud percibida por las pacientes con SOP, solo el 55\% de ellas buscan ayuda profesional para la resolución de sus síntomas somáticos, pues otras consideran que los síntomas no merecen la suficiente atención, o identifican una pobre oferta de servicios de salud especializados y eficientes (11). En principio, para algunas pacientes, incrementar la percepción de riesgo puede contribuir a un aumento en la tasa de ejecución de conductas protectoras. El cambio de estilos de vida, en conjunto con una alta automotivación y autoimagen, potencia la reducción de riesgos metabólicos y psicosociales, como también mejora sus indicadores en salud sexual y reproductiva (7). Sin embargo, las intervenciones de este corte deben realizarse con precaución, dado que si estos indicadores son altos se reduce la motivación (32); por ende, es necesario intervenir el distrés para asegurar que dichas pacientes accedan a un adecuado manejo de la dieta y de la medicación (34). Una forma útil para la reducción del estrés y de los indicadores fisiológicos relacionados podría 
ser el Mindfulness (11), que aunque reporta buenos resultados en el bienestar percibido, necesita de más estudios que confirmen la posible generalización de dicho abordaje terapéutico a las pacientes con SOP. El establecimiento de programas dietarios con y sin acompañamiento de ejercicio aeróbico, contribuye a la reducción del peso, al aumento de un estado de ánimo funcional, y al mejoramiento de los indicadores de calidad de vida percibida en las pacientes con SOP (52). Por otra parte, el establecimiento de ofertas de acompañamiento psicosocial permite que las pacientes cuenten con espacios de manifestación de sus emociones, fortalecimiento de sus estrategias de afrontamiento a las situaciones asociadas a la enfermedad misma, y creación de vínculos sociales, todas ellas alternativas clave en el incremento de la autoimagen y la autoestima. De manera adicional, es importante incluir el fortalecimiento del autocontrol, por ejemplo, ante la ingesta de sustancias no avaladas por la comunidad médica, o de la posología sugerida (10), y realizar programas educativos, para que las pacientes elijan deliberadamente entre las diferentes opciones terapéuticas disponibles (14).

Por último, es importante realizar acompañamiento a familiares, de manera que comprendan la situación y sirvan de apoyo material y emocional ante las crisis emocionales que la paciente pueda manifestar. El fortalecimiento de dichos vínculos posiblemente contribuya a evitar sensaciones de infelicidad, frustración y depresión (16), e incremente los niveles de optimismo, autoestima y bienestar social percibido. Sin embargo, el fortalecimiento del soporte social también debe hacerse con cautela, pues aunque contribuye a aspectos como la comprensión de la situación por otros pares, la construcción de lazos de confianza con los profesionales, la toma de decisiones frente a la participación en determinado tratamiento, y el ajuste en el manejo de los síntomas (53), en algunos individuos potencia una excesiva dependencia al grupo de apoyo, conllevando a un desapoderamiento hacia sus estilos de vida, en particular cuando las experiencias de los demás son negativas, lo cual les genera falta de identificación y desesperanza ante el tratamiento, y perciban que sus necesidades no son satisfechas al participar de estas comunidades (54).

\section{CONCLUSIONES}

Conocer las características psicológicas de las mujeres afectadas por el SOP es crucial para el direccionamiento de intervenciones preventivas y terapéuticas orientadas alcambio sostenido en sus estilos de vida, y por esta vía, al mejoramiento de la condición somática y mental (7). El apoyo psicológico complementa las acciones médicas, y juntas constituyen una forma más efectiva e integral de abordaje de las pacientes con SOP (32), que incide de manera positiva en su calidad de vida y bienestar. Sin embargo, es necesario seguir desarrollando investigaciones que permitan enriquecer el acervo científico al respecto, incluyendo el análisis de las variables propias de la atención en salud para las pacientes con SOP en el contexto latinoamericano.

\section{REFERENCIAS}

1. Teede H, Deeks A, Moran L. Polycystic ovary syndrome: a complex conditions with psychological, reproductive and metabolic manifestations that impacts on health across the lifespam. BMC Medicine, 2010;8:41.

2. Hergüner $S$, Harmanci $H$, Hergüner $A$, Toy $H$. Autistic traits in women polycystic ovary syndrome. Res Autism Spectr Disord 2012;6:1019-22.

3. Neinstein L, Gordon C, Katzman D, Rosen D, Woods E. Handbook of adolescent health care. Philadelphia, PA: Wolters Kluwer 2009;476:696-705.

4. Builes CA, Díaz I, Castañeda J, Pérez LE. Caracterización clínica y bioquímica de la mujer con síndrome del ovario poliquístico. Rev Col Obstet Ginecol 2006;57(1):36-44.

5. Amini L, Valian K, Avvalshahr HS, Montaeri A. Selfconfidence in women with and without polycystic ovary syndrome. 2014. J Fam Reprod Health;8(3):113-6.

6. Benson S, Janssen O, Hahn S, Tan S, Dietz T, Mann $\mathrm{K}$, et al. Obesity, depression and chronic low-grade inflammation in women with polycystic ovary syndrome. Brain Behav Immun 2008;22(2):177-84.

7. Moran L, Gibson-Helm M, Teede H, Deeks A. Polycystic ovary syndrome: a biopsychosocial understanding in young women to improve knowledge and treatment options. J Psychosom Obst Gynecol 2012;31(1):2431.

8. Bishop SC, Basch S, Futterweit W. Polycystic ovary syndrome, depression, and affective disorders. Endocr Pract 2009;15(5):475-82.

9. Dowdy A. Emotional need of teens with polycystic ovary syndrome. J Pediat Nurs 2012;27:55-64.

10. WHO. Mental health aspects of women's reproductive health. Geneva: World Health Organization; 2009.

11. Benson S, Arck PC, Tan S, Hahn S, Mann K, Rifaie N, et al. Disturbed stress responses in women with polycystic ovary syndrome. Psychoneuroendocrinology 2009;34(5):727-35.

12. Farrell K, Antoni MH. Insulin resistance, obesity, inflammation, and depression in polycystic ovary syndrome: biobehavioral mechanisms and interventions. Fertil Steril 2010;94(5):1565-74.

13. Barry JA Kuczmierczyk AR Hardiman PJ. Anxiety and depression in polycystic ovary syndrome: a systematic review and meta-analysis. Hum Reprod 2013;(26)9:2442-51.

14. Snyder BS. Polycystic ovary syndrome (PCOS) in the adolescent patient: recommendations for practice. Pediat Nursing 2005;31(5);416-21.

15. Kumapareli VL, Seneviratne RA, Wijeyaratne CN. Health-related quality of life and psychological distress in polycystic ovary syndrome: a hidden facet in South Asian women. BJOG 2011;118(3):319-28.

16. Benson S, Hahn S, Tan S, Mann K, Janssen OE, Schedlowski M, Elsenbruch S. Prevalence and implications of anxiety in polycystic ovary syndrome: results 
of an internet-based survey in Germany. Hum Reprod 2009;24(6):1446-51.

17. Masson $M$, Norström $\mathrm{K}$, Holte J, Landin-Wilhelmsen $\mathrm{K}$, Dahlgren E, Landen M. Sexuality and psychological wellbeing in women with polycystic ovary syndrome compared with healthy controls. Eur J Obstet Gyn Reprod Biol 2011;155(2):161-5.

18. Stowall D, Scriver JL, Clayton AH, Willams CD, Pastore LM. Sexual function in women with polycystic ovary syndrome. J Sex Med 2012;9(1):224-30.

19. Raja-Khan N, Agito K, Shah J, Stetter CM, Gustafson TS, Socolow H, Kunselman AR, Reibel DK, Legro RS. Mindfulness-based stress reduction for overweight/ obese women with and without polycystic ovary syndrome: Design and methods of a pilot randomized controlled trial. Contemp Clin Trials 2015;41:287-97.

20. Jones GL, Hall JM, Balen AH, Ledger WL. Health-related quality of life measurement in women with polycystic ovary syndrome: a systematic review. Hum Reprod Update 2008;14(1):15-25.

21. Kozica S, Deeks A, Gibson-Helm M, Teede H, Moran $\mathrm{L}$. Health related behaviors in women with life-style related diseases. Behav Med 2012;38(3):65-73.

22. Açmaz G, Albayrak E, Açmaz B, Baser M, Soyak M, Zararsiz, et al. Level of anxiety, depression, self-esteem, social anxiety, and quality of life among the women with polycystic ovary syndrome. Sc World J 2013. Disponible en: http://dx.doi.org/10.1155/2013/851815.

23. Pastore LM, Patrie JT, Morris WL, Dalat $P$, Bray MJ. Depression symptoms and body dissatisfaction association among polycystic ovary syndrome women. $\mathrm{J}$ Psychosom Res 2011;71(4):270-6.

24. Komarowska $H$, Stangierski A, Warmuz-Stangierska I, Ochmanska K, Wasko R, et al. Differences in the psychological and hormonal presentation of lean and obese patients with polycystic ovary syndrome. Neuro Endocrinol Lett 2013;34(7):669-74.

25. Micskei O, Deli T, Jakab A, Bugán A. Body image and quality of life in women with polycystic ovary syndrome. Orv Hetil 2014;155 (27):1071-7.

26. Bazanganipour F, Ziaei S,Montazeri A, Foroozanfard F, Kazemnejad A, Faghihzadeh S. Predictive factors of health-related quality of life in patients with polycystic ovary syndrome: a structural equation modeling approach. Fertil Steril 2013;100(5):1389-96.

27. Livadas S Chaskou S, Kandaraki A, Skourietos G, Econocmou F, Christou M, et al. Anxiety is associated with hormonal and metabolic profile in women with polycystic ovarian syndrome. Clin Endocrinol 2011;75(5):698-703.

28. Karacan E, Caglar GS, Gürsoy AY, Yilmaz MB. Body satisfaction and eating attitudes among girls and young women with and without polycystic ovary syndrome. J Pediatr Adolesc Gynecol 2014;27(2):72-7.

29. Drosdzol A, Skrzypulec V, Mazur B, Pawlinska-Chamara R. Quality of life and marital sexual satisfaction in women with polycystic ovary syndrome. Folia Histochem Cytobiol 2007;45(1):93-7.

30. Bishop SC, Basch S, Futterweit W. Polycystic ovary syndrome, depression, and affective disorders. Endocr Pract 2009;15(5):475-82.

31. Kerchner A, Lester W, Stuart SP, Dokras A. Risk of depression and other mental health disorders in women with polycystic ovary syndrome: a longitudinal study. Fertil Steril 2009;91(1):207-12.
32. Neinstein L, Gordon C, Katzman D, Rosen D, Woods E. Handbook of adolescent health care. Philadelphia, PA: Wolters Kluwer, 2009; 476-8, 696-705.

33. Hahn S, Janssen OE, Tan S, Pleger K, Mann K, Schedlowski M, Kimmig R, et al. Clinical and psychological correlates of quality of life in polycystic ovary syndrome. Eur J Endocrin 2005;153(6):853-60.

34. Kozica SL, Gibson-Helm ME, Teede HJ, Moran LJ. Assessing self-efficacy and self-help methods in women with and without polycystic ovary syndrome. Behav Med 2013;39(3):90-6.

35. Thomson RL, Buckley JD, Lim SS, Noakes M, Clifton PM, Norman RJ, Brinkworth GD. Lifestyle management improves quality of life and depression in overweight and obese women with polycystic ovary syndrome. Fertil Steril 2010;94(5):1812-6.

36. De Niet JE, de Koning CM, Pastoor H, Duivenvoorden HJ, Valkenburg O, Ramakers MJ, Passchier $\mathrm{J}$, et al. Psycholgical well-being and search in women with polycystic ovary syndrome. Hum Reprod 2010;25(6):1497-503.

37. Zangneh FZ, Naghizadeh MM, Abedinia N, Haghollahi F, Hezarehei D. Psychological signs in patients with polycystic ovary syndrome. J Family Reprod Health 2012;6(4):145-51.

38. Morotti E, Persico N, Battaglia B, Fabbri R, Meriggiola MC, Venturoli S, Battaglia C. Body imaging and sexual behavior in lean women with polycystic ovary syndrome. J Sex Med 2013;10(11):2752-60.

39. Tan S, Hahn S, Benson S, Janseen OE, Dietz T, Kimming R, et al. Psychological implications of infertility in women with polycystic ovary syndrome. Hum Reprod 2008;23(9):2064-71.

40. Bazanganipour F, Ziaei S,Montazeri A, Foroozanfard F, Kazemnejad A, Faghihzadeh S. Psychological investigation in patients with polycystic ovary syndrome. Health Qual Life Outcomes 2013;11:141.

41. Mansson M, Holte J, Landin-Wilhemsen K, Dahlgren $\mathrm{E}$, Johansson $\mathrm{A}$, Landen $\mathrm{M}$. Women with polycystic ovary syndrome are often depressed or anxious: A case control study. Psychoneuroendocrin 2008;33(8):1132-8.

42. Barnard L, Ferriday D, Guenther N, Strauss B, Balen $A H$, Dye L. Quality of Life and psychological well being in polycystic ovary syndrome. Hum Reprod 2007;22(8):2279-86.

43. Barry JA, Hardiman PJ, Saxby BK, Kuczmierczyk A. Testosterone and mood dysfunction in women with polycystic ovarian syndrome compared to subfertile controls. J Psychosom Obstet Gynaecol 2011;32(2):10411.

44. Benson S, Arck PC, Tan S, Hahn S, Mann K, Rifaie N, et al. Disturbed stress responses in women with polycystic ovary syndrome. J Psyneurn 2009;34(5)72735.

45. Holbrey S, Coulson N. A qualitative investigation of the impact of peer to peer online support for women living with polycystic ovary syndrome. BMC Wom Health 2013;13:51.

46. Hahn S, Janssen OE, Tan S, Pleger K, Mann K, Schedlowski M, Kimmig R, et al. Clinical and psychological correlates of quality of life in polycystic ovary syndrome. Eur J Endocrinol 2005;153(6):853-60.

47. Masson M, Norström K, Holte J, Landin-Wilhelmsen K, Dahlgren E, Landen M. Sexuality and psychological 
wellbeing in women with polycystic ovary syndrome compared with healthy controls. Eur J Obstet Gynecol Reprod Biol 2011;155(2):161-5.

48. Kowalczyk R, Skrzypulec V, Lew-Starowicz Z, Nowosielski K, Grabski B, Mark W. Psychlogical gender of patients with polycystic ovary syndrome. Acta Obstet Gynecol Scand 2012;91(6):710-4.

49. Ozenli Y, Haydardedeoglu B, Micozkadioglu I, Simsek E, Bulgan E, Bagis T. Anxiety, depression and ways of coping skills by women with polycystic ovary syndrome: a controlled study. J Turkish-German GynecolAssoc 2008;9(4);190-4.

50. Cipkala-Gaffin J, Talbott EO, Song MK, Bromberger J, Wilson J. Associations between psychologic symtoms and life satisfaction in women with polycystic ovary syndrome. Womens Health 2012; 21(2):179-87.

51. Benson S, Janssen O, Hahn S, Tan S, Dietz T, Mann $\mathrm{K}$ et al. Obesity, depression and chronic low-grade inflammation in women with polycystic ovary syndrome. Brain Behav Immun 2008;22:177-84.

52. Trent M, Rich M, Austin B, Gordon C. Quality of life in adolescent girls with polycystic ovary syndrome. Arch Pediatr Adolesc Med 2002;156(6):556-60.

53. Baron-Cohen S, Lombardo MV, Auyeung B, Ashwin E, Chakrabarti B, Knickmeyer R. Why are autism spectrum conditions more prevalent in males? PLOS Biol 2011 9: e1001081.

54. Dowdy A. Emotional need of teens with polycystic ovary syndrome. J Pediat Nurs 2012;27(1):55-64. 